\title{
Mechanism of Precipitation Hardening of Magnesium-Zinc Alloys*
}

\author{
By Genjiro Mima** and Yasuzo Tanaka***
}

\begin{abstract}
The strength at room temperature of $\mathrm{Mg}-4 \mathrm{wt} \% \mathrm{Zn}$ polycrystalline alloys (grain size : about $50 \mu$ ) in various aging states has been studied by the tensile test and electron-microscope observations. It is found that this alloy is age-hardened by the following mechanism.

(1) The yield strength in the early stage of aging $\left(\right.$ pre- $\left.\beta^{\prime}\right)$ is determined by the energy increment due to the formation of interface dislocations on a new surface between precipitates and the matrix. In this stage, precipitates are cut by dislocations on the basal plane.

(2) The yield strength in the intermediate stage of aging $\left(\beta^{\prime}+\beta\right)$ is determined by the energy increment due to the disturbance of atomic arrangement of the particles by dislocations passing through the particles.

(3) The yield strength in the stage of over-aging is due to the by-pass of dislocations as the Orowan's mechanism.

(4) The work hardening of the age-hardened alloys is explained by the Ashby's theory of the second slip. (Received March 17, 1971)
\end{abstract}

\section{Introduction}

Some of investigations ${ }^{(1) \sim(3)}$ on the aging sequence of magnesium-zinc alloys have elucidated that the strength of this alloy depends on the precipitation state of the transition phase $e^{(4) \sim(6)}$. But very few studies have been concerned with the strengthening mechanism of magnesium-zinc alloys are very few. Especially the study of the strengthening parameters in this alloy system has scarcely been done. Clark ${ }^{(4)}$ reported that the precipitation strengthening in this alloy conformed to the Orowan model, in which the slip dislocations bowed between particles. However, Chun et al. ${ }^{(7)}$ suggested that another strengthening mechanism was possible by the change of grain size or aging conditions. The present authors have indicated that the precipitation hardening of polycrystalline magnesium-zinc alloys should be investigated on the basis of the size and volume fraction of the precipitates ${ }^{(8)}$.

Relating to the strengthening mechanism, these different views may arise from the lack of profound understanding of the strengthening parameters. For the yield strength of this alloy, the following parameters are considered : the crystal structure and deformation mode, the kind and coherency of precipitates, the size and volume fraction of precipitates, the testing temperature, the strain rate, and so on. The strength of alloys is also affected by the work hardening due to deformation. Since the effect of grain size on the strength has already been discussed ${ }^{(8)}$, the grain size of tensile specimens in the present work is fixed at about $50 \mu$. The tensile temperature is also restricted to the vicinity of room temperature.

* This paper was originally published in Japanese in J. Japan Inst. Metals, 35 (1971), 345.

** Faculty of Engineering, Osaka University, Suita. Present address : Department of Metallurgy, Faculty of Engineering, Kansai University, Suita, Osaka, Japan.

*** Graduate School, Osaka University, Suita, Osaka. Present address : Central Research Laboratory, The Furukawa Electric Company, Limited, Shinagawa-ku, Tokyo, Japan.

(1) G. Mima and Y. Tanaka : J. Japan Inst. Metals, 33(1969), 796.
The purposes of the present work are to examine the strengthening parameters on the basis of results from the tensile test and the electron microscopic observation, and to investigate the mechanism of precipitation hardening in magnesium-zinc alloys with the same grain size and at constant testing temperature.

\section{Experimental Procedure}

For the preparation of specimens, magnesium of 99.98 wt $\%$ purity and zinc of $99.99 \mathrm{wt} \%$ purity were melted in a crucible under flux and cast into an iron mold The chemical compositions of alloys are shown in Table 1.

Table 1 Chemical composition of specimens (wt $\%$ ).

\begin{tabular}{c|c|c|c|c|c}
\hline \hline Alloy & $\mathrm{Zn}$ & $\mathrm{Si}$ & $\mathrm{Fe}$ & $\mathrm{Al}$ & $\mathrm{Mg}$ \\
\hline $\mathrm{Z} 40-1$ & 3.88 & 0.02 & 0.001 & 0.0004 & balance \\
$\mathrm{Z} 40-2$ & 4.16 & 0.026 & 0.007 & $\mathrm{tr}$ & balance \\
\hline \hline
\end{tabular}

The ingots were homogenized at $360^{\circ} \mathrm{C}$ for $48 \mathrm{hr}$ in an argon circulation furnace and water cooled. Then, the ingots were hot-forged at $300^{\circ} \mathrm{C}$, hot-rolled at about $300^{\circ} \mathrm{C}$, and cold-rolled down to about $1.5 \mathrm{~mm}$ thickness as the tensile specimens, and further rolled down to about $0.2 \mathrm{~mm}$ thickness as the thin foil specimens. The tensile specimens, whose direction was parallel to the rolling direction, were cut from the plates. The tensile specimens were $4 \mathrm{~mm}$ wide, about one $\mathrm{mm}$ in thickness and $15 \mathrm{~mm}$ in gauge-length.

After chemical polishing with a solution of $33 \mathrm{vol} \%$ nitric acid and $67 \mathrm{vol} \%$ alcohol, the tensile specimens were annealed at $360^{\circ} \mathrm{C}$ for $32 \mathrm{hr}$ under argon

(2) Y. Murakami, O. Kawano and H. Tamura : Kyoto Univ., Memoirs of the Faculty of Engng., 24 (1962), 93.

(3) L. Sturkey and J. B. Clark : J. Inst. Metals, 88 (1959 60), 77.

(4) J. B. Clark : Acta Met., 13 (1965), 1281.

(5) E. O. Hall : J. Inst. Metals, 96 (1968), 21

(6) G. Mima and Y. Tanaka : J. Japan Inst. Metals, 34 (1970), 209.

(7) J. S. Chun and J. G. Byrne : Phil. Mag., 20 (1969), 291.

(8) G. Mima and Y. Tanaka : J. Japan Inst. Metals, 35 (1971), 339. 
atmosphere and quenched into iced water. Then, in the isothermal oil bath the specimens were aged at $150^{\circ}$ and $200^{\circ} \mathrm{C}$ for a specific time. At room temperature, the tensile test was performed on the Instron tensile machine and Shimazu Autograph $1 \mathrm{~S}-5000$, the strain rate being $5.5 \times 10^{-4} \mathrm{sec}^{-1}$.

Electron microscopic observations of thin foil specimens, which were heat treated and deformed in the same way as the tensile specimens, were carried out by the electron microscope operated at $100 \mathrm{kV}$. The thin foil specimens were prepared by the controlled chemical polishing using a ethyl-niterl solution and electro-polishing using a solution of $60 \mathrm{vol} \%$ methyl alcohol, $30 \mathrm{vol} \%$ glycerin and $10 \mathrm{vol} \%$ nitric acid under the same electrolytic conditions as in the previous work ${ }^{(1)}$.

\section{Experimental Results and Discussion}

\section{Strengthening parameters}

First, let us consider the deformation mode in this alloy. In materials of the hexagonal close-packed lattice structure $(c / a \cong 1.6)$ such as this alloy, the basal slip preferentially operates as the primary slip system and the operation of other slip systems is very difficult. However, in the polycrystalline material, the operation of five independent slip systems is required at least in order to satisfy the von Mises condition. Thus, an additional deformation such as twinning, grain shearing, kinking and duplex slip plays an important role in this alloys $^{(9)(10)}$. The present author ${ }^{(11)}$ and Chun et al. ${ }^{(7)}$ have made it clear that the deformation twinning in the vicinity of room temperature can substantially be inhibited under the pressence of precipitates. Therefore, the effect of twinning can be excluded from the present study. Since the grain boundary shearing at room temperature is relatively small in pure magnesium ${ }^{(12)}$ and it becomes much smaller with the increase of zinc addition $^{(13)}$, the effect of grain boundary shearing may also be excluded, and the kinking which is said to take place incidental to the deformation twinning and the grain boundary shearing ${ }^{(12)}$ may hardly be observed. The duplex slip such as the pencil slip was observed under such a particular stress condition such as in the vicinity of grain boundary ${ }^{(8)}$. But the pencil slip is changed into the small wavy slip with aging time. Thus, the deformation mode in a relatively prolonged stage of aging is generally represented by the basal slip.

The kind of precipitates and its coherency were already reported by the present authors ${ }^{(1)}$. Namely, precipitates in magnesium-zinc alloys are the transition phase $\beta^{\prime}$ and the equilibrium phase $\beta$. The transition phase precipitates in a direction perpendicular to the basal plane and is possibly semi-coherent with the matrix. On the other

(9) B. J. Jaoul : Etude de la Plasticité et Application aux Métaux, trans. S. Morozumi and H. Hunakubo, Maruzen, (1969).

(10) A. R. Causey and E. Techtsoonian : Met. Trans., 1 (1970), 1177.

(11) G. Mima and Y. Tanaka : unpublished work.

(12) F. E. Hauser, P. R. London and J.E. Dorn : Trans. ASM, 47 (1955), 102; ibid., $48(1956), 986$.

(13) M. W. Toaz and E. J. Ripling : J. Metals, 8 (1956), 936. hand, the non-coherent plate-like equilibrium phase precipitates on the basal plane.

The size and volume fraction of precipitates are important strengthening parameters in the precipitation alloys. Since the rod-like transition phase is perpendicular to the basal plane of the matrix, the strengthening of an alloy with the transition phase may depend largely on the shape and size of precipitates on the basal plane of the matrix. Figure 1 shows the change in size of the precipitates on the basal plane for the

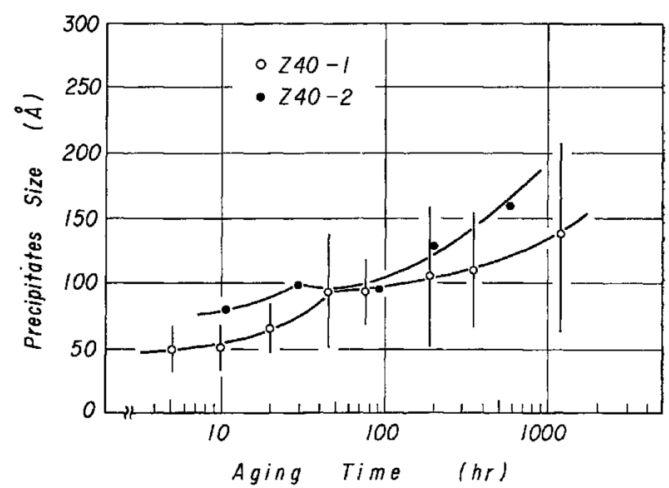

Fig. 1 The change in precipitates size on the basal plane for aged specimens at $150^{\circ} \mathrm{C}$.

specimens aged at $150^{\circ} \mathrm{C}$. It is considered that the deviation of the measuring points is based on the difference of growth rate of precipitates and shape of the cross section of precipitates on the basal plane. Although the shape of the cross section of precipitates on the basal plane of the matrix is rectangular or elliptical (as shown in Photo. 2), it may be circular at a rough estimate, because when the precipitates is polygonal on the slip plane the plastic resistance is nearly equal to that of round precipitates when the size of precipitates is not very large. Therefore, the size of precipitates on the basal plane may be represented by the mean values of a large and small particles, the long and short sides of the rectangle, and the long and short axes of the elliptic. On the above assumption, the relation between precipitates and dislocations is schematically shown in Fig. 2, and Tyson's equation (1) can be applied ${ }^{(14)}$ :

$$
\lambda=d-\pi r / 2
$$

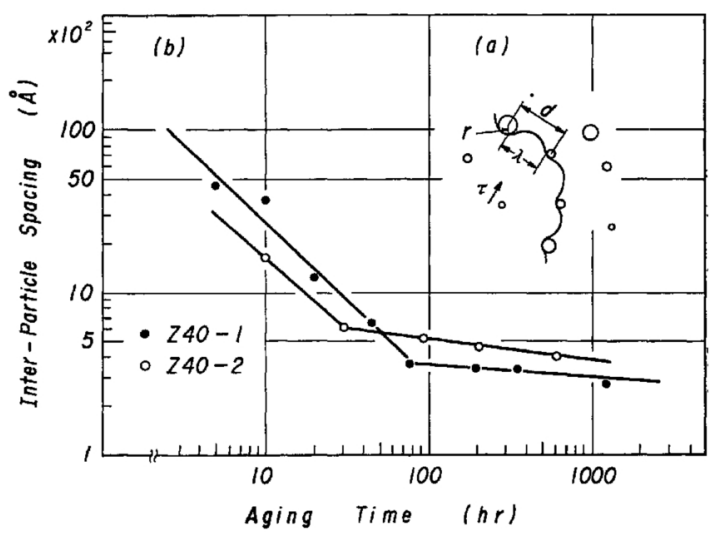

Fig. 2 The change in interparticle distance with aging time.

(14) W. R. Tyson : Acta Met, 11(1963), 61. 
where $\lambda$ is the interparticle distance, $d$ is the distance between the centers of particles and $r$ is the mean radius of particles. Figure 2 shows the relationship between the interparticle distance on the basal plane and the aging time, $\lambda$ according to the above equation. The by-pass mechanism of the Orowan type can be estimated by this interparticle distance ${ }^{(15)}$. For another mechanism, the volume fraction of precipitates serves as the strengthening parameter. According to Fullman ${ }^{(16)}$, the volume fraction of rod- or plate-shaped precipitates can be expressed as follows :

$$
f=S / d^{2}
$$

$\left\{\right.$ for the rod-shaped precipitates $S=2 \pi r^{2}$

for the plate-shaped precipitates $S=2 r t$.

Here $f$ is the volume fraction of precipitates, $d$ is the distance between the centers of precipitates, and $r$ is the mean radius of precipitates on the basal plane assuming that the shape of plate-like precipitates on the basal plane is nearly circular, and $t$ is the thickness of particles. The changes in the volume fraction of the transition phase $\beta^{\prime}$ (rod-shaped) and the equilibrium phase $\beta$ (plateshaped) are shown in Fig. 3 on the basis of eq. (2). It is shown that the volume fraction of the transition phase becomes approximately constant and that of the equilibrium phase successively increases.

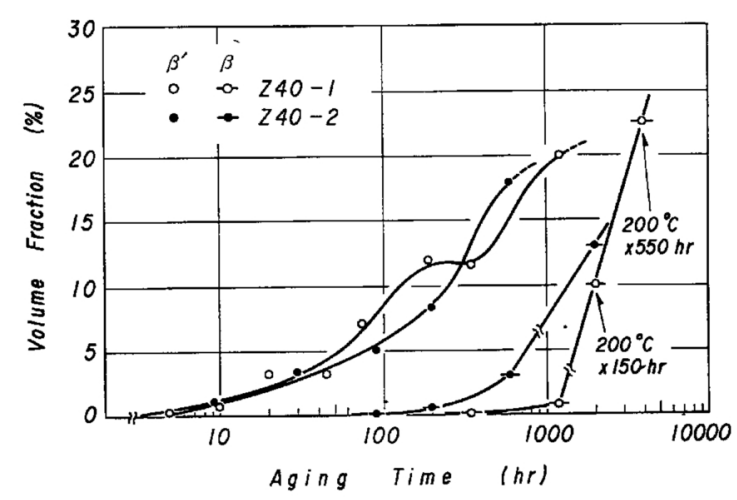

Fig. 3 The volume fraction of transition or equilibrium phase for aged specimens at $150^{\circ} \mathrm{C}$.

Generally, the shear modulus changes with the decomposition of the supersatulated solid solution. According to the estimation of the shear modulus from the initial slope of the stress-strain curve in this alloy, it has been established that the shear modulus increases with aging time and decreases upon over-aging ${ }^{(17)}$. It has also been confirmed that the shear modulus and the yield stress show a different dependence on the testing temperature as described later ${ }^{(17)}$. At room temperature, the temperature dependence of yield stress is similar to that of the shear modulus in the precipitates-free stage and the initial precipitation stage, but the temperature dependence of yield stress is larger than that of the shear modulus in the precipitation stage. It seems possible therefore that the yielding mechanism is the

(15) E. Orowan : Symposium on Internal Stress in Metals and Alloys, Inst. Metals, (1948), 451.

(16) R. L. Fullman : J. Metals, (Trans. AIME, vol. 197), 5 (1953), 477.

(17) G. Mima and Y. Tanaka : unpublished work. cutting mechanism in the initial stage of precipitation and the Orowan's by-pass mechanism or the cross-slip mechanism in the stage of over-aging ${ }^{(18)}$.

\section{Yield strength}

Since the coherency of precipitates and the matrix in this alloy is partly coherent or non-coherent, it is considered that the effect of coherency stress on the yield strength as reported by Mott-Nabarro ${ }^{(19)}$ and Gerold ${ }^{(20)}$ is not very large. Therefore, as a possible yielding mechanism, the Orowan's by-pass mechanism ${ }^{(15)}$ and the short-range interactions between the dislocation and the precipitates such as the cutting mechanism ${ }^{(21)}$ may be taken into consideration. Further, in view of the above strengthening parameters, it is the cutting mechanism should be applicable when the section size of precipitates on the basal plane is relatively small, and the by-pass mechanism when the size of precipitates is fairly large.

\section{(1) Cutting mechanism}

When a dislocation penetrates the precipitates on the basal plane as shown in Fig. 2, the following energy increment due to the cutting of the precipitates may be considered $^{(21)}:$ (a) the increase in energy, $E_{p}$, due to the formation of phase boundary in internally ordered particles, (b) the increase in energy, $E_{s}$, due to the formation of the new surface between the precipitate and the matrix, (c) the increase in energy, $E_{i}$, due to the formation of the surface dislocation, and (d) the increase in energy, $E_{d}$, due to the formation of jog or dislocation dipoles resulting from the difference of the slip plane in the matrix and the precipitates. $E_{a}$ of them may be excluded because the slip plane in the matrix (basal) and the precipitates (prismatic) are parallel. As for the other parameters, $E_{p}$ is roughly estimated at about $10^{-18}$ $\mathrm{kg} \cdot \mathrm{cm}, E_{s}$ at about $10^{-29} \mathrm{~kg} \cdot \mathrm{cm}$ and $E_{i}$ at about $10^{-19}$ $\mathrm{kg} \cdot \mathrm{cm}$. Thus, it is shown that the increase in energy due to the cutting depends largely on $E_{p}$.

When the interparticle spacing on the glide plane is $\lambda$, the work done by the applied stress is equal to $\tau b^{2} \lambda$ when the dislocations are cutting through the particle as far as the Burgers vector $b$. Then, this work is nearly equal to $E_{p}$ in this alloy. On the other hand, when the interface energy of $\gamma_{p}$ per unit area is formed in the internally ordered particles, the increase in energy $E_{p}$ due to the cutting of the particles is expressed as

$$
E_{p}=t b \gamma_{p},
$$

where $t$ is the length of the dislocation segment in the precipitates. Therefore, the increase in applied stress necessary to move the dislocation can be expressed as

$$
\Delta \tau=t \gamma_{p} /(b \lambda) \text {. }
$$

Figure 4 shows the change in yield stress for the $\mathrm{Z} 40$ alloy at $150^{\circ} \mathrm{C}$. The change in yield stress for

(18) T. Miyazaki : Bull. Japan Inst. Metals, 8 (1969), 204.

(19) N. F. Mott and F. R. N. Nabarro : Proc. Phys. Soc., 52 (1940), 86.

(20) V. Gerold : Acta Met., 16(1968), 823.

(21) A. Kelly and R. B. Nicholson : Progress Mat. Sci., Pergamon Press, Vol. 10, (1963). 


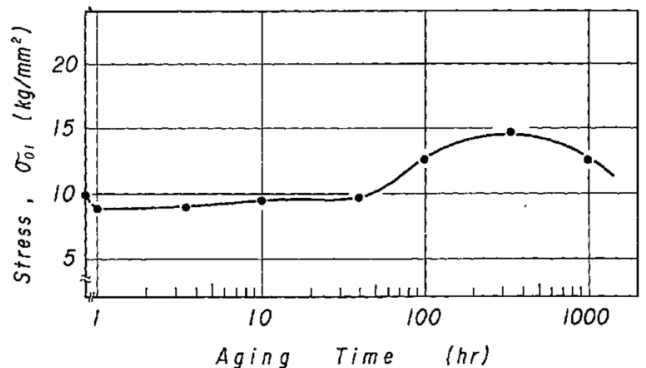

Fig. 4 The aging curve of $\mathrm{Mg}-4 \mathrm{wt} \% \mathrm{Zn}$ alloy aged at $150^{\circ} \mathrm{C}$ for yield stress.

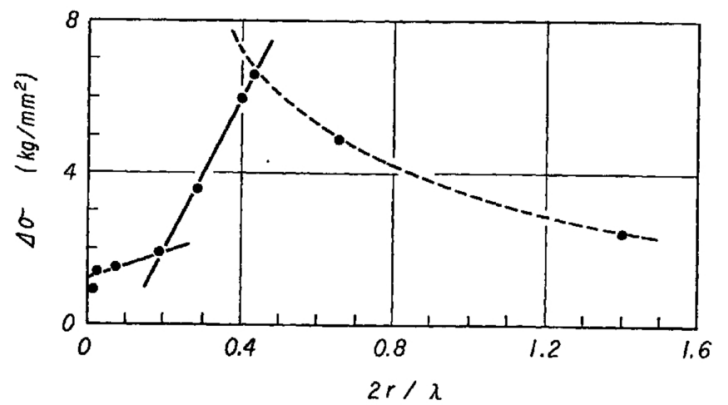

Fig. 5 Plot of the increment of yield stress against $t / \lambda$ for $\mathrm{Mg}-4 \mathrm{wt} \% \mathrm{Zn}$ alloy aged at $150^{\circ} \mathrm{C}, 2 r \simeq t$.

$t / \lambda\left(\Delta \sigma=\sigma_{H}-\sigma_{Q}, \sigma_{H}\right.$ is the yield strength of aged specimens and $\sigma_{Q}$ is the yield strength of quenched specimens) is linear in the $t / \lambda$ range of 0.2 to 0.5 as shown in Fig. 5, obeying eq. (4). It is essential for estimation of the quantity $\gamma_{p}$ in eq. (4) to assume that in this alloy the transition phase $\left(\mathrm{MgZn}^{\prime}\right)$ internally forms as the $\mathrm{AB}$ type superlattice. According to Williams et al. ${ }^{(22)(23)}, \gamma_{p}$ for the precipitates of this type can be expressed as follows:

$$
r_{p}=\frac{K S^{2} \Delta E}{a_{0}^{2} N}
$$

Where $K$ is constant $(=1.5 \sim 2.5), S$ is the long range order parameter $(=0.7 \sim 1.0), a_{0}$ is the lattice parameter, $N$ is the Avogadro's number, and $\Delta E$ is the ordering energy which is equal to $R T_{c} / 2$ where $R$ is the gas constant and $T_{c}$ is the ordering temperature. The calculated values of $\gamma_{p}$ are $10 \sim 30 \mathrm{erg} / \mathrm{cm}^{2}$ and then the stress increment of $1 \sim 5 \mathrm{~kg} / \mathrm{mm}^{2}$ due to the cutting is given by substituting them and the experimental values into eq. (4). Further, considering the Taylor's index, the results agrees well with the yield strength as shown in Fig. 5. The lower range of $t / \lambda$ of 0.2 corresponds to the formation of the pre- $\beta^{\prime}$ whose structure possibly differs from that of the transition phase.

Assuming that the number of $\mathrm{Mg} / \mathrm{Zn}$ pairs in the pre- $\beta^{\prime}$ is smaller than that in the transition phase and the structure of the pre- $\beta^{\prime}$ is hardly disordered by the dislocation crossing the slip plane, the increase in energy due to the cutting will be estimated by $E_{i}$ rather than $E_{p}$. The increase in yield stress due to $E_{i}$ is expressed as

$$
\Delta \tau=\frac{G \cdot \Delta b^{2}}{2 \pi(1-\nu)} \cdot \ln \frac{R}{r_{0}} \cdot \frac{1}{b \lambda},
$$

(22) R. O. Williams : Acta Met., 5(1957), 241.

(23) F. C. Nix and W. Shockley : Rev. Mod. Phys., 10 (1938), 1. where $G$ is the shear modulus, $\Delta b$ is the magnitude of Burgers vector of the interface dislocation, $\nu$ is the Poisson's ratio, $R$ is the precipitates diameter (nearly equal to $t$ ) and $r_{0}$ is the core size of dislocation (nearly equal to $b$ ). Substituting various values into eq. (6), the increment of yield strength is approximately equal to $0.25 \mathrm{~kg} / \mathrm{mm}^{2}$, and this is corresponding to $\Delta \sigma$ of about $1 \mathrm{~kg} / \mathrm{mm}^{2}$. The above results is in good agreement with Fig. 5.

Thus, it is found that the yield strength in the earlier stage of aging (pre- $\beta^{\prime}$ ) is governed by the interface of the precipitates and the matrix, and the yield strength in the middle of aging is governed by the internal structure of precipitates, but the yield strength in the latter stage of aging does not conform to the eq. (4).

\section{(2) The by-pass mechanism}

There are two mechanism to account for the yield stress of a material containing particles which are not sheared by the dislocations moving on the slip plane : the Orowan's by-pass mechanism(15) and the cross-slip mechanism $^{(24)}$.

The latter mechanism will not be able to occur considering with the temperature dependence of the shear modulus and the yield stress, the relationship between the particle size and the interparticle distance, and the difficulty of the extending of the dislocations on the basal plane and the prismatic plane.

Therefore, this mechanism does not directly govern the yield stress without the special condition such as the grain boundary.

According to Orowan, the additional flow stress due to the precipitation is expressed by $\Delta \tau=2 T /(b \lambda)$. For the line tension, $T$, Ashby ${ }^{(25)}$ and Formean et al. ${ }^{(26)}$ minutely argued, and the additional flow stress due to the by-pass was given by :

$$
\begin{aligned}
& \Delta \tau_{e}=\frac{1}{1.18} \cdot \frac{2 A^{\prime}}{b \lambda} \text { for the edge dislocation } \\
& \Delta \tau_{s}=\frac{1}{1.18} \cdot \frac{1}{1-\nu} \cdot \frac{2 A^{\prime}}{b \lambda} \text { for the screw dislocation } \\
& A^{\prime}=\frac{G b^{2}}{4 \pi} \cdot \ln \frac{r}{b}
\end{aligned}
$$

where $G$ is the shear modulus of the matrix, $b$ is the magnitude of the Burgers vector, $\nu$ is the Poisson's ratio and $r$ is the radius of precipitates. Substituting various values for eq. (7), the results as shown in Table 2

Table 2 The calculated values of Orowan's stressincrement of $\mathrm{Mg}-4 \mathrm{wt} \% \mathrm{Zn}$ alloy $(\mathrm{kg} / \mathrm{m})$.

\begin{tabular}{l|c|c|c}
\hline & $150^{\circ} \mathrm{C} \times 350 \mathrm{hr}$ & $150^{\circ} \mathrm{C} \times 1200 \mathrm{hr}$ & $200^{\circ} \mathrm{C} \times 550 \mathrm{hr}$ \\
\hline $\begin{array}{l}\left(\beta^{\prime}+\beta\right) / / c \text { axis } \\
\beta \_c \text { axis }\end{array}$ & 6.1 & 5.7 & 0.72 \\
& - & 0.24 & 2.1 \\
\hline $\begin{array}{l}\text { Total stress- } \\
\text { increment }\end{array}$ & 6.1 & 5.9 & 2.8 \\
\hline
\end{tabular}

(24) M. Ashby : Z. Metallk., 55 (1964), 5.

(25) M. F. Ashby: Office of Naval Research Tec. Rep., No. 515 (1966).

(26) A. J. K. Formean and M. J. Makin : Phil. Mag., 14 (1966), 911. 
correspond to the transition phase and the equilibrium phase. Since the calculated value in Table 2 is for the edge dislocation, it becomes much larger for the screw dislocation. Considering the Taylor's index for the calculated value in Table 2 , the calculated values are by far larger than the measured ones. This may be ascribed to the difference in the-shear moduli of the precipitates and the matrix with the aging time ${ }^{(24)(27)}$, and it must be considered for the corrected stress in the Orowan theory. Taking this into consideration for this alloy would make the calculated values nearly equal to the measured ones.

\section{Work hardening}

The work hardening will already take place in polycrystalline alloys on macroyielding. Thus, the work hardening must be discussed with the yield strength in order to explain the strength of the polycrystalline precipitation alloy.

Figure 6 shows the work hardening curves in the Z40 alloy. It is shown that the work hardening is not very large in the quenched or the pre-precipitated state and is remarkably large in the precipitated state, while it becomes remarkably large in the precipitation state and becomes small in the over-aged state.

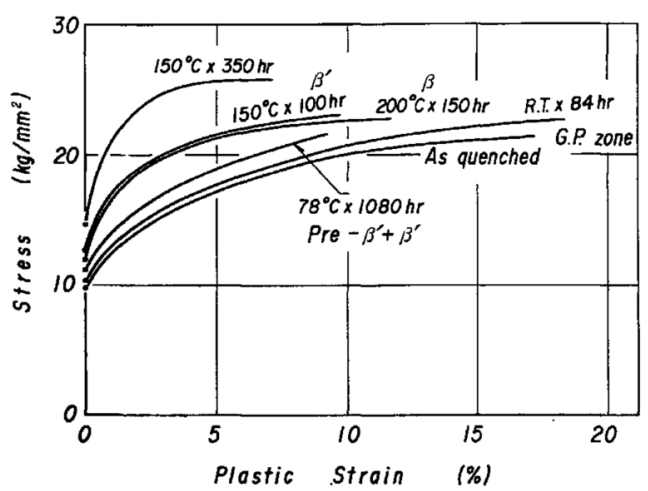

Fig. 6 The work hardening eurve of $\mathrm{Mg}-4 \mathrm{wt} \% \mathrm{Zn}$ alloy.

A theory to explain the work hardening has been established by Fisher, Hart and Pry ${ }^{(28)}$. In this theory the dislocation moves leaving the dislocation loop around the particles, which can be applied to the single crystal containing the precipitates whose volume fraction is rather small and to the range of plastic strain below about $2 \%$. But, as this alloy is polycrystalline, the theory of secondary slip proposed by Ashby et al. ${ }^{(29)}$ may be applied because this theory can be extended to a higher range of plastic strain. When the hard particle suffers the homogeneous shearing, the stress increment around the particles is relieved by the secondary deformation. Consequently, the glide dislocation on the secondary slip plane and the prismatic loops around the particles are created. Since the primary slip plane is perpendicular to the secondary slip plane in this alloy,

(27) R. L. Fleisher : Acta Met., 9(1961), 996.

(28) J. C. Fisher, E. W. Hart and R. H. Pry : Acta Met., 1(1953), 336.

(29) R. Ebeling and M. F. Ashby : Phil. Mag., 13 (1965), 805; ibid., 14(1966), 1157. the glide dislocation due to the secondary slip will appear predominantly. Thus, it is presumed that the work hardening arises from the interaction between the primary dislocation and the glide due to the secondary slip. The flow stress in the region of work hardening is introduced by Ashby et al. as given by

$$
\tau-\tau_{0}=K G \sqrt{\frac{b f \alpha}{2 r}},
$$

where $\tau$ is the flow stress, $\tau_{0}$ is the yield stress, $G$ is the shear modulus of the matrix, $b$ is the magnitude of the Burgers vector, $f$ is the volume fraction of precipitates, $\alpha$ is the shear strain, $r$ is the radius of particles and $K$ is the constant due to the kind of dislocation $(=0.2 \sim$ 0.4). Figure 7 shows the plots of $\frac{\sigma-\sigma_{0}}{G}$ and $\sqrt{\frac{b f \alpha}{2 r}}$ following the eq. (8), where plastic strains of $1,2,5$, $10 \%$ are taken, assuming that its value corresponds to a 1.4-fold shear strain. It is established from the linearity of the plot that the secondary slip model is applied to the explanation of work hardening.

Although the slope in Fig. 7 is 2.6 for the pre- $\beta^{\prime}$ and 0.9 for the $\left(\beta^{\prime}+\beta\right)$ precipitates, this slope is nearly equal to the theoretical values of $0.2 \sim 0.4$ when the Taylor's index is taken into consideration.

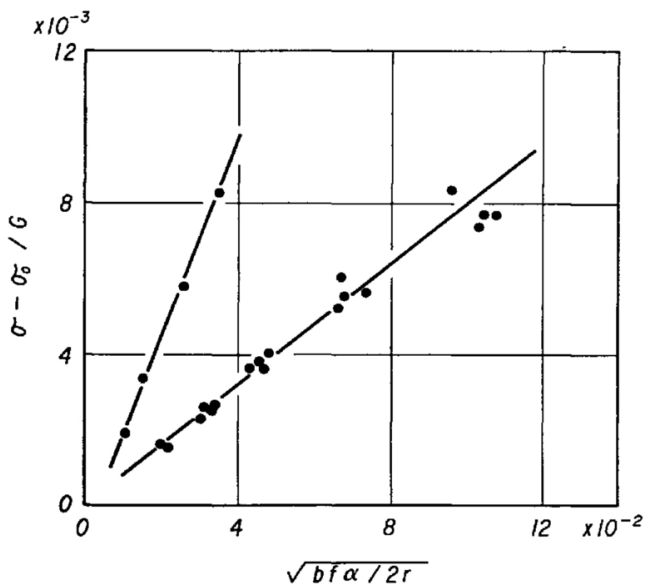

Fig. 7 Correlation of the increment of flow stress due to strain hardening with the predictions of the Ashby's theory for $\mathrm{Mg}-4 \mathrm{wt} \% \mathrm{Zn}$ alloy, $2 r \simeq t$.

\section{Deformed structures of precipitated alloys}

To confirm the mechanism of precipitation hardening, electron microscopic observations are carried out.

Photo. 1 shows an electron micrograph of Z 40 alloy deformed up to few pct after aging at $150^{\circ} \mathrm{C}$ for $600 \mathrm{hr}$. It is shown that the dislocations are obstructed by the precipitates and are bowing out among the precipitates and cutting them.

Photo. 2 shows an electron micrograph of $\mathrm{Z} 40$ alloy deformed up to few pet after aging at $200^{\circ} \mathrm{C}$ for $150 \mathrm{hr}$. Dislocation loops around the large precipitates on the basal plane and tangled dislocations around the other precipitates are observed. It is evident that the specimens in the latter stage of aging are work-hardened by the dislocation loops and tangled dislocations. 


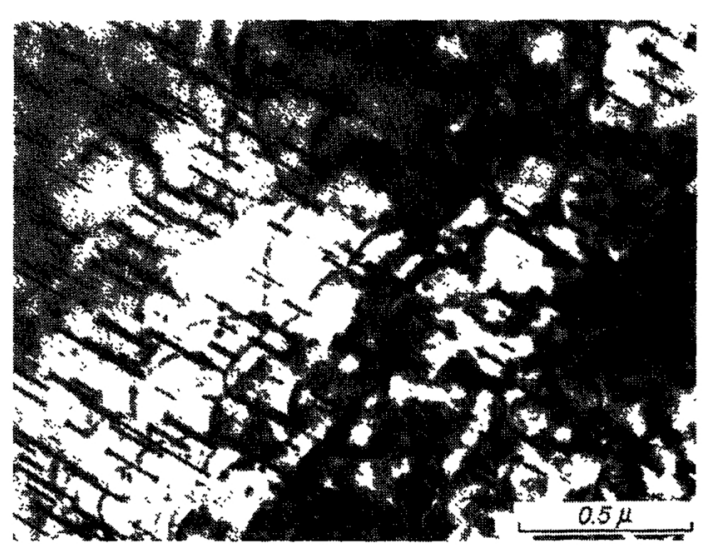

Photo. 1 The electron micrograph. of $\mathrm{Mg}-4 \mathrm{wt} \% \mathrm{Zn}$ alloy aged at $150^{\circ} \mathrm{C}$ for $600 \mathrm{hr}$ and deformed few pct.

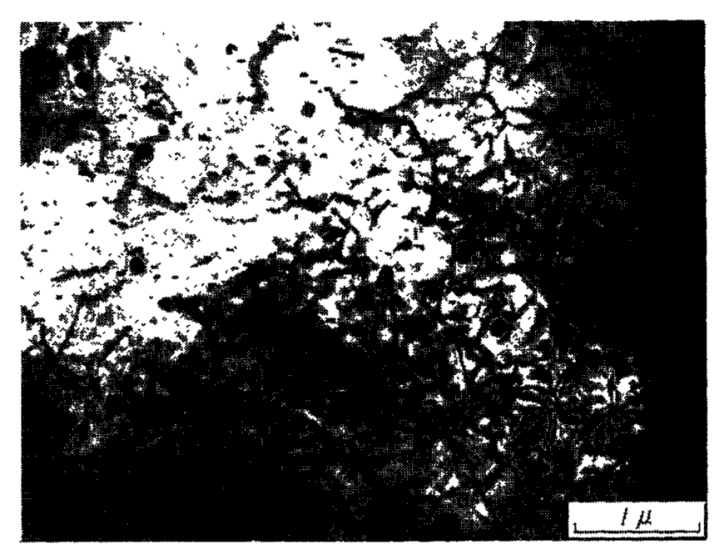

Photo. 2 The electron micrograph of $\mathrm{Mg}-4 \mathrm{wt} \% \mathrm{Zn}$ alloy aged at $200^{\circ} \mathrm{C}$ for $150 \mathrm{hr}$ and deformed few pct.

\section{Conclusions}

The strength of $\mathrm{Mg}-4 \mathrm{wt} \% \mathrm{Zn}$ polycrystalline (about $50 \mu$ ) alloy at room temperature was studied by the tensile test and electron microscopic observation. It was found that this alloy is age-hardened by the following mechanism.

(1) The yield strength in the early stage of aging $\left(\right.$ pre- $\left.\beta^{\prime}\right)$ is determined by the increase in energy which is required to make an interface dislocation between the precipitates and the matrix in consequence of cutting of particles by the dislocations.

(2) The yield strength in the intermediate stage of aging $\left(\beta^{\prime}+\beta\right)$ is determined by the increase in energy due to the disturbance of atomic arrangement of the particles by the dislocations passing through the particles.

(3) The yield strength in the stage of over-aging is due to the by-pass of dislocations based on the Orowan's mechanism.

(4) The work hardening in this alloy can be explained by the Ashby's theory of the secondary slip.

\section{Acknowledgment}

The authors acknowledge their indebtedness to Prof. Dr. Toshimi Yamane for his advice and suggestions. 